\title{
A Design Plan of a Game System including an Automatic Narrative Generation Mechanism: The Entire Structure and the World Settings
}

\author{
Jumpei Ono \\ Graduate School of Software and Information Science, Iwate Prefectural University \\ 152-52, Sugo, Takizawa, Iwate 020-0693, Japan \\ Takashi Ogata \\ Faculty of Software and Information Science, Iwate Prefectural University \\ 152-52, Sugo, Takizawa, Iwate 020-0693, Japan \\ E-mail: g236m001@s.iwate-pu.ac.jp,t-ogata@iwate-pu.ac.jp
}

\begin{abstract}
In this paper, we propose a narrative generation system based on the scenario generation method in tabletop roleplaying games (TRPGs). This system uses an integrated narrative generation system (INGS) that synthesizes various narrative generation mechanisms. The TRPG is an analog game in which one or more players (PLs) play the role of characters within the framework of the narrative prepared by the game master (GM). A PL promotes the development of scenes, which leads to the completion of the narrative. In particular, we concentrate on the "world setting" that consists of the rules or constricts for a narrative and also the components defined by the rules and the stage setting of the narrative. In an ordinary scenario, a GM sets a basic world setting for the narrative generation through the interaction between the PLs. We consider preliminarily prepared scripts corresponding to various world settings in addition to the detailed mechanism to use it.
\end{abstract}

Keywords: integrated narrative generation system, narrative generation, table-top role playing game, world setting

\section{Introduction}

We design a narrative generation system based on a game format that refers to a scenario generation method in tabletop role-playing games (TRPGs) ${ }^{1}$. This system is an application of the integrated narrative generation system (INGS) ${ }^{2-4}$ that was developed using the game method; the proposed system generates a narrative based on the interactions between a user and the system.

The INGS consists of a concept generation mechanism to generate a structure of narrative, and a surface representation mechanism to represent the narrative through sounds, words and pictures. The INGS has noun conceptual dictionary and verb conceptual dictionary for constructing an event concept in the narrative ${ }^{5}$, story content knowledge for constituting the structure of the narrative ${ }^{6}$, each event concept in the conceptual structure of narrative is connected in front state and behind it by a state-event translation knowledge base ${ }^{4}$.

\section{Overview of the TRPG}

The TRPG is an analog game where a player (PL) becomes a character and plays each role within the framework of a narrative prepared by the game master (GM). This role-playing by the character reaches a conclusion as the scene develops, and as a result, a big narrative is completed.

Further, Fig. 1 shows the relationship between the concepts of a "scenario." The "scenario" consists of the 
"world setting" and the "scene sequence. The "scenario" is formed during the progression of a game through the interaction between the GM and a PL.

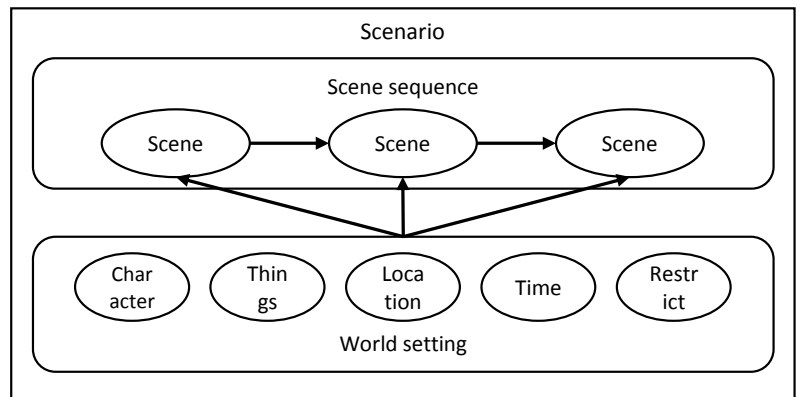

Fig. 1 Scenario and its components

We will now discuss the TRPG in detail. First, a "scene sequence" in the "scenario" is created based on the actual incident or fictitious it. Next, the "scene sequence" by the GM is modified and expanded as the game progresses. The "world setting" includes the setting of the game such as the place and time and consists of the limitations or rules applicable to the "scenario."

\section{Generation Process}

The generation process is divided into three steps"preparation of necessary information," "generation of narrative," and "output for a conclusive result.

In the "Preparation of necessary information" step implies the preparations required to obtain the information for an initial scenario prepared by the GM. The user inputs the information that is necessary for the GM to generate the initial state of the world setting and the scene sequence. Based on the "world setting", the user inputs the editing contents of the conceptual dictionary, as described. The user can operate the limitations of a verb. For example, if the user adds "a cat" to the limitations of a verb concept "buy," a cat becomes to be able to "buy" a thing like a human.

By using the initial scene sequence, a user inputs an event that will play a key role in the scenario; for example, "a man obtains a treasure." An initial scene sequence will consist of elements. This scene will be a partial structure of a story expressed in a conceptual form. It consists of states indicating the static information of characters, things, locations, events indicating the dynamic change in a state, and the relationships among them that can be used to connect these events structurally. The narrative is a structure consisting of events and relationships in the INGS. This is called an attribute frame. It records the properties and characteristics of individual elements and is connected to an event through an ID.

In the "generation of narrative" step, the scenario was fleshed out of the scene sequence based on the exchanges between the GM and the PL. Therefore, this part is the core of the system.

On the basis of a scene shown by the GM, the PL suggests a scene. The scene chain expansion utilizes the story (story contents) generation function of the INGS. The extended scene sequence is shown as a scenes sequence to the PL by the above mentioned process. When a progress condition is met in the present person, attribute frame of a thing, the place after this processing, GM stop that GM receives the suggestion of the scene from PL temporarily and show a "scene (climax)" and a "scene (ending)".

In the "Output of a conclusive result" refers to the stage that outputs the scenario information in the final stage, expanding the scene sequence one way or another. For example, a novel-like sentence is thought to be created and produced as output. In this case, the game process is equivalent to a novel generation process. The system outputs a sentence as shown in the generation process. A structured text is obtained by compiling the sentences outputted for every scene. The user includes more names in the scenes and completes a story by modifying the outputted end results.

\section{World Setting}

This section shows a conceptual dictionary in the INGS, and describes the "world setting".

\subsection{Conceptual dictionary in the INGS}

The conceptual dictionary is related to the "world setting". The conceptual dictionary is used for controlling the world setting in a designed system. The conceptual dictionary includes a noun conceptual dictionary, verb conceptual dictionary, and modifier conceptual dictionary (conceptual dictionary for adjective, adjective verb, and adverb); it stores knowledge at the conceptual level as components. A 
characteristic function in verb conceptual dictionary is to describe the "restriction" while using the elements in events such as " $\sim$ do $\sim$." To adjust this "restriction," the system should be able to modify a realistic event such as "Taro eats an ice cream" into an unrealistic event such as "The car eats an ice cream."

\subsection{Elements in the World Setting}

The "world setting" is like a stage for the progression of a narrative. The "world setting", define the type of characters, things, locations, time, event and action that characters can perform. As a similar example, Nomura suggested the "background world". The "background world" consists of "space", "existence", "time", and the component generates "phenomenon" and "event".

Here, we mention each element in the "world setting".

\section{A) Restrictions}

The "world setting" including the stage setting of the games such as places or times, has a kind of limitation in a "scenario." The narrative could include "a human cannot fly in the sky without a vehicle" in the realistic "world setting." On the other hand, "a person can fly in the sky" in the fantastic "world setting." In this way, the restriction is a kind of a rule for the action possibility.

\section{B) Locations}

"Locations" is the space where characters or things exist and, for the objective achievement of a character, it can be either a beneficial or an unfavorable element. They show the environment of the stage for the narrative. For example, a character is police, climate etc. in the realistic "locations".

\section{C) Time}

"Time" is the time when characters or things exist and affects the styles of characters or things. For example, for the case "to light," the character of the reality world uses a lighter, whereas a more primitive world character uses a firestone.

\section{D) Characters}

"Characters" are all the people appearing in the narrative, which include the characters controlled by the user. Each character has the purpose, in the case of a character being controlled by a PL, and the PL defines the purpose of the character as the game progresses; other characters are defined by the GM at the start of the game. Those purpose is stored an attribute frames in the states.

\section{E) Things}

"Things" represent the tools that a character uses; particularly these are the elements that are necessary for achieving the purpose of the character.

The above five elements are the "world setting" component. When the designed system generates a narrative and progresses game, the elements is required relationship between characters, things, locations, that are included in the "world setting". Concretely, there is (1) a relationship among characters, things, and locations, (2) an ability to achieve a certain purpose, (3) a motive of each character. In addition, for (2), the restrictions in the "word setting" limit the ability of each element.

\subsection{Generation of a Narrative through the World Setting}

Fig.2, Fig.3 and Fig.4 show (1) a relationship among characters, things, and locations (Fig.2), (2) an ability to achieve a certain purpose (Fig.3), (3) a motive of each character (Fig.3).

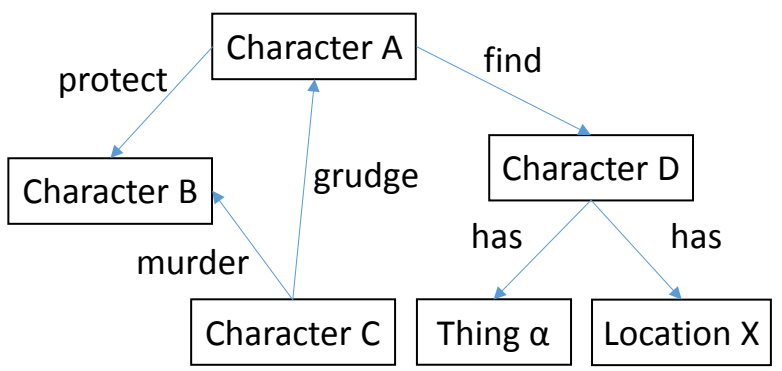

Fig. 2 (1) Relationship among characters, things, and locations

"Character A" can search [any person]
"Character A" can fight against [any person]
"Character B" dies of a [disease]
"Character C" can interfere with "Character A"
"Character C" can fight against [any person]
"Character D" can cure [disease] using "thing $\alpha "$ at
"location X"

Fig. 3 (2) Ability to achieve a certain purpose 
"Character A" wants to help "Character B"

"Character C" wants to disturb "Character A"

"Character C" wants to kill "Character B"

"Character D" wants money

Fig. 4 (3) Motive of each character

The system generates a "scene sequence" shown in Fig. 5 by using the relationship (Fig. 3) and motive (Fig. 4). The "Character $C$ " inserts " $\mathrm{C}$ interferes with $\mathrm{A}$ " into behind either scene for to achieve its own purpose.

Scene 1. "B" gets a [disease] $\rightarrow$

Scene 2. "A" searches for a doctor $\rightarrow$

Scene 3. "A" hears the wish of "D" $\rightarrow$

Scene 4. "A" grants the wish of "D" $\rightarrow$

Scene 5. "D" starts the treatment of " $B$ " $\rightarrow$

Scene 6-a. "B" is saved, or

Scene 6-b. "B" is not saved

Fig. 5 Generated scene sequence

The system gives an appearance based on the world setting in Fig. 2, as shown in Fig. 5. The appearances of each element are change based on "world setting". For example, in the realistic "world setting," the "character $A$ " is a detective and the "character $C$ " is a climate. In contrast, in the fantastic "world setting," the "character $\mathrm{A}$ " is hero, "[disease]" is a curse, and the "character C" is a devil.

\section{Conclusion}

In this paper, we proposed a narrative generation system based on a game format that refers to the scenario generation method in a TRPG. This system can support story generation through the interactions with the user.

In the present conditions, the system design assumes that a human being controls the PL side and generates a story accordingly. When a system computer controls the PL side, there is a possibility of generation of a story that is unexpected for a human user. However, in the present conditions, the scene for the PL side is decided by the user and the procedure for this generation is not clear. Therefore, the scene is difficult to be designed by an automated PL. In future, we would like to automate the process of scene generation on the PL side.

\section{References}

1. "Tabletop role-playing game - Wikipedia, the free encyclopedia",

https://en.wikipedia.org/wiki/Tabletop_roleplaying_game (Last access, 2015, July).

2. T. Ogata and A. Kanai, An Introduction of Informatics of Narratology: on Thought and Technology of Narrative Generation (Gakubunsha, Tokyo, 2010). (In Japanese)

3. T. Akimoto and T. Ogata, An Information Design of Narratology: The Use of Three Literary Theories in a Narrative Generation System, The International Journal of Visual Design, 7(3) (2014) 31-61.

4. T. Ogata, Development of the Integrated Narrative Generation System, Proc. of the 13th Forum on Information Technology (2), (2014) 323-330. (In Japanese)

5. T. Ogata, Building Conceptual Dictionaries for an Integrated Narrative Generation System, Journal of Robotics, Networking and Artificial Life, 1(4) (2015) 270-284

6. T. Ogata and J. Ono, Language Notation Dictionaries and the Use in an Integrated Narrative Generation System, IEICE Technical Report, 115(69) (2015) 25-30. (In Japanese)

7. K. Nomura, Sory Sekai no Tsukurikata (How to Making a Story World) (Shinkigensya, Tokyo, 2014). (In Japanese) 